\title{
Use of Featforward Mobile Phone App Associated with Decreased Cardiometabolic Risk Factors in Patients with Chronic Conditions
}

Sara B Golas ${ }^{1}$, MA; Ramya Palacholla ${ }^{1,2,3}$, MD, MPH; Amanda Centi ${ }^{1}$, PhD; Odeta Dyrmishi ${ }^{1}$, MPA; Stephen Agboola $^{1,2,3}$; Joseph Kvedar ${ }^{1,2,3}$, MD; Kamal Jethwani ${ }^{1,2,3}$, MD

${ }^{1}$ Partners Connected Health Innovation, Boston, MA, United States

${ }^{2}$ Massachusetts General Hospital, Boston, MA, United States

${ }^{3}$ Harvard Medical School, Boston, MA, United States

Corresponding Author:

Sara B Golas, MA

Partners Connected Health Innovation

25 New Chardon

Boston, MA,

United States

Phone: 6439956

Email: sgolas@partners.org

\section{Abstract}

Background: Physical inactivity is one of the leading risk factors contributing to rising rates of chronic diseases and has been associated with deleterious health outcomes in patients with chronic disease conditions. FeatForward is a mobile phone app designed to encourage patients with cardiometabolic risk (CMR) factors to increase their levels of physical activity.

Objective: To evaluate the effect of the FeatForward mobile phone app on physical activity levels (primary outcome) and global CMR factors (secondary outcomes) in patients with chronic conditions.

Methods: In this 6-month, 2-arm randomized controlled trial, adult participants endorsing at least 1 study-eligible condition (obesity, [pre-]diabetes, [pre-]hypertension) were enrolled and assigned to either the intervention group (FeatForward app and standard care) or control group (standard care only). The primary and secondary outcomes were, respectively, change from baseline in physical activity (step count) and CMR factors (weight, body mass index [BMI], waist circumference, glycated hemoglobin $\left[\mathrm{HbA}_{1 \mathrm{c}}\right]$, fasting blood glucose, systolic/diastolic blood pressures, serum lipids, C-reactive protein [CRP]). CMR data were collected at 3 time-points: baseline, 3 months, and 6 months. Step count data were recorded continuously by patients' study-issued activity trackers and collected in batches at 3 and 6 months. At study end, patients' weekly average step counts (WAS) were calculated as total steps taken divided by days of step data (0-7) for each of 26 study weeks. Mixed-effects linear regression models evaluated change over time between groups for the primary outcome and secondary outcomes. All models controlled for baseline values. The step count model additionally controlled for proportion of days without data, defined as (7days of data) / 7. Analyses were conducted for both groups overall, and by disease cohort (obesity, diabetes, hypertension).

Results: Step count and CMR data were analyzed for 128 intervention and 133 control patients. There were no demographic differences between groups. While there was an overall downward trend in WAS for both groups, the intervention group decreased significantly less than the control group, with a slope of -29.3 steps per week compared to controls' $-57.9(P=.02)$. Intervention patients with obesity slightly increased their step count overtime, differing significantly from controls (slope of 0.9 vs -90.2 ; $P<.001$ ). Intervention patients significantly lowered their BMI per study month compared to controls (slopes -0.23 vs -0.02 ; $P=.04)$. Additionally, intervention patients with hypertension significantly decreased weight $(P=.003)$, BMI $(P=.002)$, and CRP $(P=.03)$ per month compared to the control group. Waist circumference, $\mathrm{HbA}_{1 \mathrm{c}}$, fasting blood glucose, blood pressure, and lipids did not differ significantly by group or disease cohort over time.

Conclusions: While it is common for patient engagement with physical activity trackers to decrease over the course of a study, patients using the FeatFoward app had a slower decline in physical activity compared to controls. Intervention patients experienced a reduction in their BMI from a mean of 34.3 to 33.4 , compared to controls' 34.8 to 35.0. Patients with hypertension experienced significant decreases in BMI, weight, and CRP compared to controls. Future analyses will evaluate the impact of app engagement levels on step counts and CMR factors for the intervention group.

(iproc 2018;4(2):e11882) doi: $\underline{10.2196 / 11882}$ 


\section{KEYWORDS}

physical activity; chronic disease; mixed-effects linear regression

Edited by T Hale; this is a non-peer-reviewed article. Submitted 09.08.18; accepted 29.08.18; published 17.09.18.

Please cite as:

Golas SB, Palacholla R, Centi A, Dyrmishi O, Agboola S, Kvedar J, Jethwani K

Use of Featforward Mobile Phone App Associated with Decreased Cardiometabolic Risk Factors in Patients with Chronic Conditions iproc 2018;4(2):e11882

URL: http://www.iproc.org/2018/2/e11882/

doi: $\underline{10.2196 / 11882}$

PMID:

(C) Sara B Golas, Ramya Palacholla, Amanda Centi, Odeta Dyrmishi, Stephen Agboola, Joseph Kvedar, Kamal Jethwani. Originally published in Iproceedings (http://www.iproc.org), 17.09.2018. This is an open-access article distributed under the terms of the Creative Commons Attribution License (https://creativecommons.org/licenses/by/4.0/), which permits unrestricted use, distribution, and reproduction in any medium, provided the original work, first published in Iproceedings, is properly cited. The complete bibliographic information, a link to the original publication on http://www.iproc.org/, as well as this copyright and license information must be included. 